\title{
Les mouvements sociaux et la transition progressiste sous un régime autoritaire
}

\author{
VUONG Thu Trang \\ Ecole doctorale \\ Sciences Po Paris \\ 27 Rue Saint-Guillaume, 75007 Paris, France \\ Email: thutrang.vuong@sciencespo.fr
}

Ce brouillon: la 2ème version

Paris, 16 Octobre 2019

Ce travail s'inspire du développement de la communauté LGBT+ au Vietnam des années récentes, plus précisément depuis la création de l'organisation VietPride et la première marche Pride à Hanoi en 2012. Après 7 ans, les marches Pride annuelles ont lieu dans de plus en plus de provinces vietnamiennes (37 provinces, au moment de l'écriture de ce texte). Si le développement de ces mouvements visant l'égalité et conformité au respect des droits de l'Homme a vu un essor à la fois en échelle et en quantité, les événements organisés par VietPride ont également suscité des réactions de plus en plus drastiques des groupes de tendances conservatrices dans la société. On observe ainsi la formation des ailes politiques, quoique uniquement sur des enjeux sociaux, dans une société jusque-là peu politisée.

L'idée principale de ce travail est d'étudier les mouvements sociaux, en particulier ceux des minorités sexuelles, sous le régime autoritaire du Vietnam. L'intérêt est d'avoir un portrait intensif des initiatives et mouvements LGBT+ au Vietnam, afin de trouver comment ceux-ci se distinguent des autres actions collectives (telles que les démonstrations vis-à-vis les conflits territoriaux dans la mer de l'Est), et évaluer son impact sur non seulement la société mais aussi le climat politique dans le grand public du Vietnam et finalement sur le régime luimême.

L'état de la littérature va traiter le sujet en trois temps. D'abord, une brève présentation des paradigmes de la sociologie des mouvements sociaux (cf. Érik Neveu) pour pouvoir plus tard mieux placer les mouvements LGBT+ au Vietnam dans son contexte et analyser des réactions contre ceux-ci. Les théories de ressources, de catnet et des relations entre organisation militante et l'établissement politique seraient d'intérêt. 
Ensuite, un compte-rendu du régime communiste au Vietnam, les traditions politiques depuis la réunification de 1975 et le système politique et la politique de gouvernement, pour éclairer la configuration politique dans laquelle n'importe quelle action collective se déroulerait. Sur cette scène marquée par d'une part un régime corrompu et d'autre part la répression de toutes critiques du régime, des mouvements sociaux se heurtent souvent à l'oppression, en particulier quand ils sont perçus comme contraires à la politique du Parti, par exemple les démonstrations antichinoises en 2014 ou bien les protestes violentes d'inspiration luddite dans les fabriques de Formosa en 2016.

Point de précision : par action collective on fait référence à des actions non-encadrées par le syndicat communiste qui n'est qu'une extension du Parti communiste autoritaire et n'ont aucun poids en tant que contre-pouvoir politique. Terminologie à retravailler et à redéfinir.

Enfin, une étude de la genèse et du développement des communautés LGBT+ et leur activisme au Vietnam. La société civile (restreinte) du Vietnam se caractérise par l'apolitisme du fait des traditions autoritaires voire totalitaires dans des années d'après-guerre (1975 dans le contexte vietnamien) jusqu'à des Réformes 1986 ; pendant cette période dite "de construction du socialisme ", l'économie et la société civile étaient comparables à celles des pays de l'Europe de l'Est avant la transition démocratique. Parler politique d'une manière sérieuse, en particulier quand il s'agit du système ou de l'organisation partisane, se perçoit encore comme un tabou. Par contre, les mouvements sociaux - en occurrence des mouvements des minorités sexuelles - qui ont bien des potentiels à redéfinir la gouvernance à travers leur combat visant à revendiquer des droits civils (et non au changement politique en soi) sont souvent tolérés, voire passivement encouragés, par l'État. On peut dire même qu'ils "passent sous le radar " (goes under the radar) du gouvernement autoritaire. Ceci est même plus intéressant si on le compare avec l'oppression LGBT+ en Chine et encore en d'autres régimes autoritaires qui se montrent plus ancrés dans des principes religieux (ce qui n'est pas le cas au Vietnam).

Les hypothèses principales portent sur deux aspects. Dans un premier temps, l'activisme et les actions collectives qui ne dérivent pas des idéologies androcentriques ou bien universalistes et qui ne visent pas directement à attaquer l'organisation du régime sont considérés par le gouvernement autoritaire comme "plus sociaux ", " moins politiques " et ainsi moins comme une menace. La tradition du matérialisme marxiste écarte et la manque d'une fondation religieuse cohérente et stricte font en sorte que des organisations religieuses n'ont pas vraiment de levier sur ce champ. Ainsi, les mouvements LGBT+ ne se heurtent pas à l'oppression étatique et ont une vraie chance de populariser/banaliser des revendications politiques dans une société qui n'y est pas habituée. Démocratisation à travers un développement de la société civile?

Dans un deuxième temps, il ne faut pas nier des pressions sociales contre ces mouvements. Alors que les soucis de moralité vis-à-vis la "déviance" sexuelle n'émergent pas (contrairement, par exemple, aux débats sur le projet de loi Sogie aux Philippines), des arguments à la fois de type naturaliste/essentialiste et traditionaliste surgissent. Ces 
arguments s'inscrivent largement dans des principes généralement conservateurs, et montrent une cohérence frappante - même dans la présentation sémantique - avec des arguments chez des " right-wing conservatives " étatsuniens ou bien des républicains/centredroits de la famille politique européenne. Politisation du société qui vient après la polarisation politique?

\section{Références}

Napier, N. K., \& Hoang, V. Q. (2013). What we see, why we worry, why we hope: Vietnam going forward. Boise ID, USA: Boise State University CCI Press.

Neveu, E. (2011). Sociologie des mouvements sociaux. Paris, France: La Découverte.

Nguyen, T. V. A., Nguyen, N. N. Q., Khuat, T. H., Nguyen, T. P. T., Do, T. T., Vu, X. T., Tran, K., Ho, M. T., Nguyen, T. H. K., Vuong, T. T., \& Vuong, Q. H. (2019). Righting the misperceptions of men having sex with men: a prerequisite for protecting and understanding gender incongruence in Vietnam. Journal of Clinical Medicine, 8(2), 105; DOI: 10.3390/jcm8010105.

Vuong, Q. H. (2014). Vietnam's political economy: a discussion on the 1986-2016 period. CEB-ULB WP. No. 14/010. Université Libre de Bruxelles.

Vuong, Q. H, Ho, T. M., Nguyen, H. T., La, V. P., Vuong, T. T., Hanh, V. T., Nguyen, M. H., \& Ho, T. M. (2019). On how religions could accidentally incite lies and violence: Folktales as a cultural transmitter. SocArXiv (26 Sept.); DOI: 10.31235/osf.io/8n3c5

Vuong, Q. H., La, V. P., Vuong, T. T., Nguyen, V. H., Ho, M. T., Nguyen, T. H. K., ... \& Ho, M. T. (2018). Cultural additivity: Behavioural insights from the interaction of Confucianism, Buddhism, and Taoism in folktales. Palgrave Communications, 4(1), 143; 143 | DOI: 10.1057/s41599-018-0189.

Vuong, T. T., Semerák, V., \& Vuong, Q. H. (2019). The Vietnamese Economy at the Crossroads. In: R. Macdonald (Ed.). Southeast Asia and the ASEAN Economic Community (pp. 91143). Cham, Switzerland: Palgrave Macmillan. DOI: 10.1007/978-3-030-19722-3_3. 